\title{
Novel Dark Matter Constraints from Antiprotons in Light of AMS-02
}

\author{
Alessandro Cuoco, ${ }^{*}$ Michael Krämer, ${ }^{\dagger}$ and Michael Korsmeier ${ }^{\dagger}$ \\ Institute for Theoretical Particle Physics and Cosmology, RWTH Aachen University, 52056 Aachen, Germany
}

(Received 24 October 2016; revised manuscript received 9 March 2017; published 9 May 2017)

\begin{abstract}
We evaluate dark matter (DM) limits from cosmic-ray antiproton observations using the recent precise AMS-02 measurements. We properly take into account cosmic-ray propagation uncertainties, fitting DM and propagation parameters at the same time and marginalizing over the latter. We find a significant indication of a DM signal for DM masses near $80 \mathrm{GeV}$, with a hadronic annihilation cross section close to the thermal value, $\langle\sigma v\rangle \approx 3 \times 10^{-26} \mathrm{~cm}^{3} \mathrm{~s}^{-1}$. Intriguingly, this signal is compatible with the DM interpretation of the Galactic center gamma-ray excess. Confirmation of the signal will require a more accurate study of the systematic uncertainties, i.e., the antiproton production cross section, and the modeling of the effect of solar modulation. Interpreting the AMS-02 data in terms of upper limits on hadronic DM annihilation, we obtain strong constraints excluding a thermal annihilation cross section for DM masses below about $50 \mathrm{GeV}$ and in the range between approximately 150 and $500 \mathrm{GeV}$, even for conservative propagation scenarios. Except for the range around $\sim 80 \mathrm{GeV}$, our limits are a factor of $\sim 4$ stronger than the limits from gamma-ray observations of dwarf galaxies.
\end{abstract}

DOI: 10.1103/PhysRevLett.118.191102

Introduction.-Cosmic-ray (CR) antiprotons are a powerful tool to investigate the particle nature of dark matter (DM); see, for example, [1-13]. DM constraints from CRs are, however, affected by uncertainties in the description of CR propagation in the Galaxy. Thus, CR DM limits have so far been derived for benchmark propagation models, like the $\mathrm{min} / \mathrm{med} / \mathrm{max}$ scenarios [2] obtained from observations of the boron over carbon $(\mathrm{B} / \mathrm{C})$ ratio. Such benchmark models introduce an order-of-magnitude uncertainty in the DM interpretation of CR fluxes.

The antiproton CR spectrum has recently been measured by the AMS-02 experiment with high precision [14]. It is thus timely to evaluate the antiproton DM constraints in light of the new data. We will improve on previous analyses in two crucial aspects: First, the new AMS-02 data allow us to significantly reduce the uncertainties in the $\mathrm{CR}$ propagation. Although B/C data from AMS-02 have been recently published [15], there is, however, evidence that the propagation of heavy nuclei like $\mathrm{B}$ and $\mathrm{C}$ is different from the propagation of light nuclei like $p$ and $\bar{p}$ [16] (but see also [17-19]). Thus, using $\mathrm{B} / \mathrm{C}$ data to constrain $\mathrm{CR}$ propagation is likely to introduce a bias when analyzing antiprotons. We will instead follow the analysis of Ref. [20] (hereafter KC16) and use the measured $\bar{p}$ flux to directly constrain the propagation scenario, thus avoiding any bias. In addition, as a second important new feature, we will constrain CR propagation including a potential $\bar{p}$ flux from DM annihilation. Previous analyses have, in contrast, assumed a certain propagation scenario (or a small number of fixed benchmark scenarios) and, thus, a fixed antiproton background to then constrain a DM contribution in a second step (although see [11] for an improved approach). Here, with a joint DM and CR propagation analysis, we will, for the first time, explore possible correlations and degeneracies between the two components, providing more robust and reliable DM constraints.

Dark matter.-Dark matter annihilation in the Galaxy leads to a flux of antiprotons from the fragmentation of standard model (SM) particles. The corresponding source term can be written as

$$
q_{\bar{p}}^{(\mathrm{DM})}\left(\boldsymbol{x}, E_{\mathrm{kin}}\right)=\frac{1}{2}\left(\frac{\rho(\boldsymbol{x})}{m_{\mathrm{DM}}}\right)^{2} \sum_{f}\langle\sigma v\rangle_{f} \frac{d N_{\bar{p}}^{f}}{d E_{\mathrm{kin}}},
$$

where $m_{\mathrm{DM}}$ is the DM mass and $\rho(\boldsymbol{x})$ the DM density profile. Furthermore, $\langle\sigma v\rangle_{f}$ denotes the thermally averaged annihilation cross section for the SM final state $f$, DM+ $\mathrm{DM} \rightarrow f+\bar{f}$, and $d N_{\bar{p}}^{f} / d E_{\text {kin }}$ the corresponding antiproton energy spectrum per DM annihilation. Note that the factor $1 / 2$ corresponds to Majorana fermion DM.

We use the Navarro-Frenk-White (NFW) DM density profile [21] $\rho_{\mathrm{NFW}}(r)=\rho_{h} r_{h} / r\left(1+r / r_{h}\right)^{-2}$, with a characteristic halo radius $r_{h}=20 \mathrm{kpc}$ and a characteristic halo density $\rho_{h}$, normalized to obtain a local DM density $\rho_{\odot}=$ $0.43 \mathrm{GeV} / \mathrm{cm}^{3}$ [22] at the solar position $r_{\odot}=8 \mathrm{kpc}$. To quantify the impact of the choice of the DM profile on our results, we will compare with the Burkert profile [23] $\rho_{\text {Bur }}(r)=\rho_{c}\left(1+r / r_{c}\right)^{-1}\left(1+r^{2} / r_{c}^{2}\right)^{-1}$, with a core radius of $r_{c}=5 \mathrm{kpc}$ and again normalized at the solar position.

The yield of antiprotons per DM annihilation and the corresponding energy distribution $d N_{\bar{p}}^{f} / d E_{\text {kin }}$ depend on the DM mass, the relevant SM annihilation channels, and the antiproton yield from the fragmentation of SM particles. We employ the results presented in Ref. [24] and focus on the annihilation into bottom quarks, DMDM $\rightarrow b \bar{b}$, for illustration. 
TABLE I. Analysis constraints on the fit parameters and their ranges of variation in the fit.

\begin{tabular}{|c|c|c|c|c|}
\hline \multicolumn{2}{|c|}{$\begin{array}{l}\text { Propagation } \\
\text { parameters }\end{array}$} & $\begin{array}{l}\text { Fit with- } \\
\text { out DM }\end{array}$ & $\begin{array}{l}\text { Standard fit } \\
\text { with DM }\end{array}$ & Fit range \\
\hline \multicolumn{2}{|l|}{$\gamma_{1, p}$} & $1.54_{-0.18}^{+0.04}$ & $1.41_{-0.01}^{+0.19}$ & $1.2-1.8$ \\
\hline \multicolumn{2}{|l|}{$\gamma_{2, p}$} & $2.425_{-0.002}^{+0.023}$ & $2.531_{-0.010}^{+0.008}$ & $2.3-2.6$ \\
\hline \multicolumn{2}{|l|}{$\gamma_{1}$} & $1.56_{-0.18}^{+0.03}$ & $1.21_{-0.02}^{+0.22}$ & $1.2-1.8$ \\
\hline \multicolumn{2}{|l|}{$\gamma_{2}$} & $2.388_{-0.003}^{+0.021}$ & $2.480_{-0.005}^{+0.005}$ & $2.3-2.6$ \\
\hline$R_{0}$ & \multirow[t]{3}{*}[\mathrm{GV}]{} & $8.43_{-1.93}^{+0.27}$ & $5.01_{-0.12}^{+1.30}$ & $1.0-10$ \\
\hline$s$ & & $0.38_{-0.01}^{+0.11}$ & $0.46_{-0.06}^{+0.01}$ & $0.05-0.9$ \\
\hline$\delta$ & & $0.361_{-0.043}^{+0.005}$ & $0.245_{-0.007}^{+0.015}$ & $0.2-0.5$ \\
\hline \multicolumn{2}{|r|}{$\left[10^{28} \mathrm{~cm}^{2} / \mathrm{s}\right]$} & $7.48_{-1.88}^{+1.52}$ & $9.84_{-2.85}^{+0.26}$ & $0.5-10.0$ \\
\hline$v_{A}$ & {$[\mathrm{~km} / \mathrm{s}]$} & $23.8_{-0.91}^{+3.09}$ & $28.5_{-0.64}^{+1.5}$ & $0-30$ \\
\hline & {$[\mathrm{km} / \mathrm{s}]$} & $26.9_{-3.33}^{+34.7}$ & $45.3_{-19.2}^{+5.69}$ & $0-100$ \\
\hline \multirow{2}{*}{$\begin{array}{l}z_{h} \\
\phi_{\mathrm{AMS}}\end{array}$} & {$[\mathrm{kpc}]$} & $6.78_{-2.70}^{+0.22}$ & $5.35_{-1.27}^{+1.65}$ & $2-7$ \\
\hline & {$[\mathrm{GV}]$} & $580_{-50}^{+65}$ & $520_{-35}^{+35}$ & $0-1.8$ \\
\hline \multicolumn{5}{|c|}{ DM parameters } \\
\hline \multicolumn{2}{|c|}{$\log \left(m_{\mathrm{DM}} / \mathrm{GeV}\right)$} & & $1.85_{-0.03}^{+0.02}$ & $1-5$ \\
\hline \multicolumn{2}{|c|}{$\log \left(\langle\sigma v\rangle / \mathrm{cm}^{3} / \mathrm{s}\right)$} & & $-25.57_{-0.03}^{+0.09}$ & $-(28-23)$ \\
\hline \multicolumn{2}{|c|}{ Experiment } & \multicolumn{3}{|c|}{$\chi^{2}$ (Number of data points) } \\
\hline \multicolumn{2}{|c|}{$p($ AMS-02) } & $9.6(61)$ & $6.2(61)$ & \\
\hline \multicolumn{2}{|c|}{$p$ (VOYAGER) } & $1.8(4)$ & $0.4(4)$ & \\
\hline \multicolumn{2}{|c|}{ He (AMS-02) } & $30.8(65)$ & $24.8(65)$ & \\
\hline \multicolumn{2}{|c|}{ He (VOYAGER) } & $2.3(4)$ & $1.6(4)$ & \\
\hline \multicolumn{2}{|c|}{$\bar{p} / p(\mathrm{AMS}-02)$} & $26.6(42)$ & $12.6(42)$ & \\
\hline \multicolumn{2}{|l|}{ Total } & $71.0(176)$ & $45.6(176)$ & \\
\hline
\end{tabular}

Analysis.-To derive predictions for the fluxes of protons, helium, and antiprotons near Earth, we solve the standard diffusion equation [25] using GALPROP [26,27]. We assume a cylindrical symmetry for our Galaxy, with a radial extension of $20 \mathrm{kpc}$. The propagation parameters which determine the shape of the injection spectrum include the spectral indices of the protons and the heavier species, $\gamma_{1, p}, \gamma_{2, p}$ and $\gamma_{1}, \gamma_{2}$, respectively, the two break positions $R_{0}$ and $R_{1}$, as well as smoothing factors $s$ and $s_{1}$. The propagation is assumed to be homogenous and isotropic. It is constrained by the normalization $D_{0}$ and slope $\delta$ of the diffusion coefficient, the velocity of Alfvén magnetic waves, $v_{A}$, connected to reacceleration, the convection velocity $v_{0 c}$, the normalization of the proton and helium fluxes, $A_{p}$ and $A_{\mathrm{He}}$, respectively, the Galaxy's half height $z_{h}$, and the solar modulation potential $\phi_{\mathrm{AMS}}$, in the framework of the force-field approximation. See Supplemental Material [28] and KC16 for more details. We also take into account the production of tertiary antiprotons [29]. The DM component of the CR flux, finally, is determined by the DM mass $m_{\mathrm{DM}}$ and the DM annihilation cross section $\langle\sigma v\rangle$, for any given choice of the DM profile and the SM annihilation channel.

We stress that this is a simplified scenario, since diffusion is likely to be nonhomogenous and anisotropic at some level. On the other hand, this simplified model has been able to explain the observations so far and has been assumed in past studies. It is thus important to address the implication of the new data within this model. A critical assessment of this base scenario will be the subject of future studies. Nonetheless, for the purposes of this analysis, we can consider the homogenous propagation coefficient $D_{0}$ as an effective parameter describing an average propagation, since we propagate only light nuclei, which share similar propagation properties. Violations of homogeneity will be manifested as a different $D_{0}$ for heavier nuclei, which have a different propagation length. We discuss this issue in more detail in the Supplemental Material [28] in relation to boron and carbon.

The above propagation and dark matter parameters are determined in a global fit of the AMS-02 proton and helium fluxes [30,31], and the AMS-02 antiproton to proton ratio [14], complemented by proton and helium data from CREAM [32] and VOYAGER [33]. The CREAM data extend to large rigidities of up to $\approx 100 \mathrm{TV}$ and allow us to determine the position $R_{1}$, amount $\Delta \gamma=\gamma_{3}-\gamma_{2}$, and smoothness $s_{1}$ of the second break in the rigidity dependence of the source. The VOYAGER data at rigidities of $\mathcal{O}(\mathrm{GV})$, on the other hand, are used to constrain the solar modulation potential $\phi_{\text {AMs }}$.

The ranges of variation of the parameters that enter our prediction of the CR flux are listed in Table I next to the fit results. Having fixed the strength and position of the second break in rigidity as in $\mathrm{KC} 16$ leaves 16 free parameters to be determined from a global fit to the AMS-02, CREAM, and VOYAGER data. We use MULTINEST [34] to scan this parameter space and derive the corresponding profile likelihoods. Details of the global fit are presented in KC16.

Results.-The result of our global fit is shown in Fig. 1 for the antiproton to proton ratio, for both the case in which DM is included (left panel) and the case without a DM component (right panel). We consider the rigidity range $R \geq 5 \mathrm{GV}$, for which the force-field approximation should describe solar modulation reliably. Adding DM annihilating into $b \bar{b}$, with $m_{\mathrm{DM}} \approx 80 \mathrm{GeV}$ and $\langle\sigma v\rangle \approx 3 \times$ $10^{-26} \mathrm{~cm}^{3} / \mathrm{s}$, results in a much better fit and provides an intriguing hint for a DM signal in the antiproton flux. The improvement of the fit quality is significant: We find a $\chi^{2} /$ (number of degrees of freedom) of $71 / 165$ for the fit without DM, which is reduced to $46 / 163$ when adding a DM component. Formally, $\Delta \chi^{2}=25$ for the two extra parameters introduced by the DM component corresponds to a significance of $\sim 4.5 \sigma$, although this does not take into account possible systematics errors.

The comparison of the two panels provides a deeper insight into the reason for the large improvement of the fit when DM is included. We can see that, without DM, the 

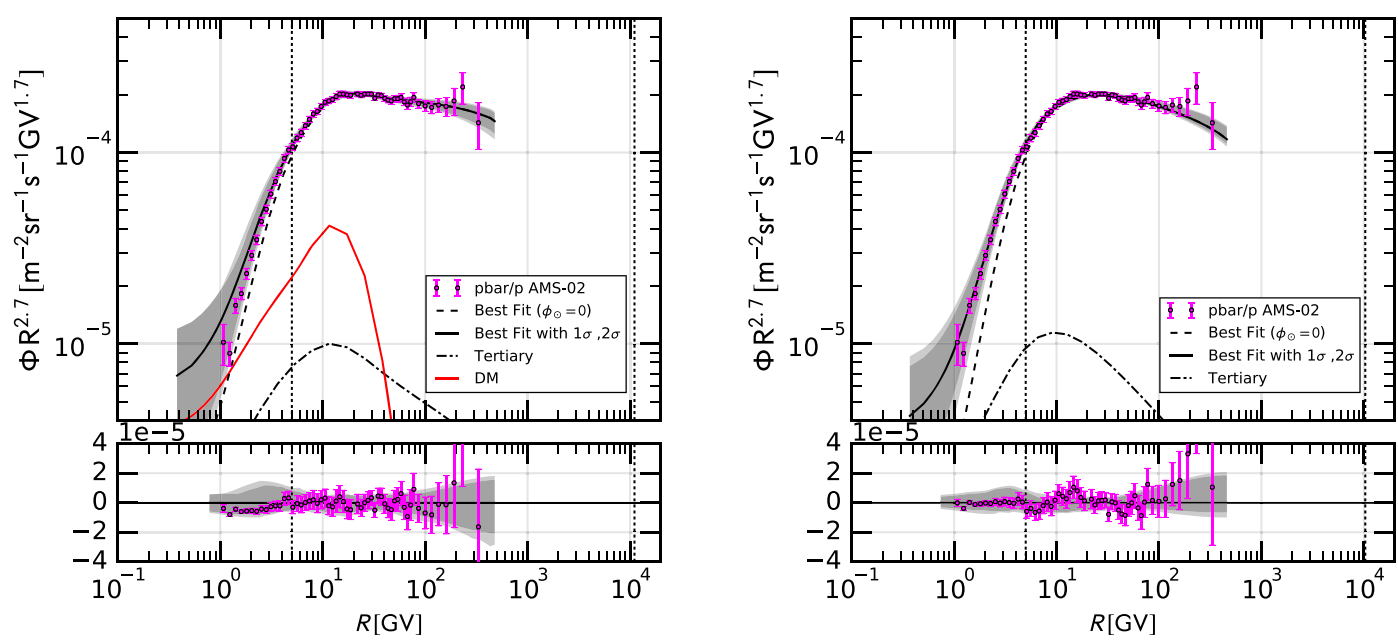

FIG. 1. Comparison of the best fit of the $\bar{p} / p$ ratio to the AMS-02 data [14], with a DM component (left panel) and without DM (right panel). The lower panels show the corresponding residuals. The fit is performed between the dotted lines, i.e., for rigidities $5 \mathrm{GV} \leq R \leq 10 \mathrm{TV}$. The gray bands around the best fit indicate the 1 and $2 \sigma$ uncertainty, respectively. The dashed black line (labeled " $\phi_{\odot}=0 \mathrm{MV}$ ") shows the best fit without a correction for solar modulation. The solid red line shows the best fit DM contribution. We also show, for comparison, the contribution from astrophysical tertiary antiprotons denoted by the dot-dashed line.

residuals show a sharp feature, similar to a break, at a rigidity of $\approx 18 \mathrm{GV}$. This feature is present in the measured spectrum and cannot be described by the secondary antiprotons only, since their predicted spectrum is too smooth compared to the data. We see instead that the DM component, shown separately in the left panel, possesses a distinctive feature which matches the structure of the residuals without DM. For comparison, we also show the contribution from background tertiaries, which peaks at similar rigidities but which cannot fit the strength and shape of the excess.

The preferred range of DM masses and annihilation cross sections is shown in Fig. 2. Intriguingly, this region is in

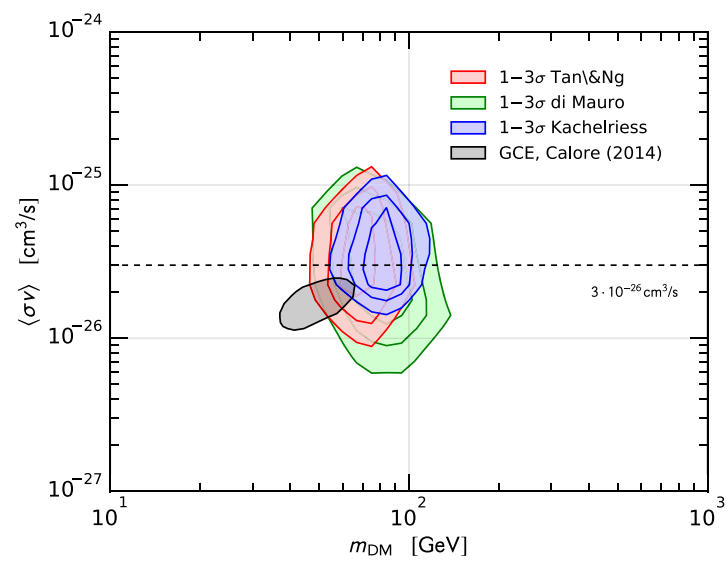

FIG. 2. Best fit regions $(1,2$, and $3 \sigma)$ for a DM component of the antiproton flux, using the antiproton cross-section models of [41] (Tan and Ng), [42] (di Mauro et al.), and [43] (Kachelriess, Moskalenko, and Ostapchenko). For comparison, we also show the best fit region of the DM interpretation of the Galactic center gamma-ray excess [39] and the thermal value of the annihilation cross section, $\langle\sigma v\rangle \approx 3 \times 10^{-26} \mathrm{~cm}^{3} \mathrm{~s}^{-1}$. very good agreement with the DM interpretation of the Galactic center gamma-ray excess [35-39]. We show for comparison the preferred DM best fit region obtained from the Galactic center gamma-ray excess in Ref. [39]. Also, a similar hint for DM has been found in Ref. [6], in relation to PAMELA antiproton data [40].

A known systematic uncertainty affecting the fit is the imperfect knowledge of the antiproton production cross section [42-47], which determines the flux of secondary antiprotons produced by the interactions of primary protons and helium nuclei on the interstellar medium gas. Adopting the recent cross-section estimates from [42,43], rather than the GALPROP default [41], does not reduce the evidence for a DM matter component in the antiproton flux and modifies only slightly the preferred ranges of DM mass and annihilation cross section; see Fig. 2. This represents an important test, since the cross sections used are quite different in nature. While those of Refs. [41,42] are based on a phenomenological parameterization of the available cross-section data, the cross section of Ref. [43] is based on a physical model implemented through Monte Carlo generators. While this check does not exhaust the range of possible systematics related to the antiproton cross section, a more robust assessment of this issue requires more accurate and comprehensive experimental antiproton cross-section measurements.

From Table I, we note that including a DM component induces a shift in some of the propagation parameters. In particular, the slope of the diffusion coefficient, $\delta$, changes by about $30 \%$ from a value of $\delta \approx 0.36$ without $\mathrm{DM}$ to $\delta \approx 0.25$ when $\mathrm{DM}$ is included. This stresses the importance of fitting at the same time DM and CR background. The changes induced by a DM component in the other CR propagation parameters are less than about $10 \%$. More details are reported in Supplemental Material [28]. 
As a further estimate of systematic uncertainties, we have extended the fit range down to a rigidity of $R=1 \mathrm{GV}$. In this case, the fit excludes a significant DM component in the antiproton flux. This can be understood from the residuals for this case, which are very similar to the ones shown in the right panel in Fig. 1. Clearly, the excess feature at $R \approx 18 \mathrm{GV}$, responsible for the DM preference in the default case, still remains. The reason why DM cannot accommodate this excess anymore is the low-rigidity tail of the DM spectrum-cf. Fig. 1 (left panel)—which would overshoot the experimental data below $5 \mathrm{GV}$. Nonetheless, although the data at $R \lesssim 5 \mathrm{GV}$ appear to disfavor a DM component in the antiproton flux, the situation is not conclusive: At rigidities $R \lesssim 5 \mathrm{GV}$, solar modulation deviates from the simple force-field approximation and exhibits also charge-dependent effects [48,49]. Thus, a deeper scrutiny of the antiproton excess and of a potential DM signal will require a dedicated study of the solar modulation below $5 \mathrm{GV}$, for which it would be desirable to have a time series of the proton and antiproton fluxes.

In the remainder of this Letter, we will make the conservative assumption of no DM detection and derive constraints on the hadronic DM annihilation cross section as a function of the DM mass. Our limits on the annihilation cross section $\langle\sigma v\rangle$ as a function of $m_{\mathrm{DM}}$ are obtained by marginalizing over the $\mathrm{CR}$ propagation uncertainties. Technically, we divide the likelihood samples in the $\langle\sigma v\rangle-m_{\text {DM }}$ plane obtained from the MULTINEST scan into 20 slices in $m_{\mathrm{DM}}$, equally spaced in $\log \left(m_{\mathrm{DM}}\right)$ between $m_{\mathrm{DM}}=10 \mathrm{GeV}$ and $100 \mathrm{TeV}$. For each $m_{\mathrm{DM}}$ slice, we derive the 1D profile likelihood as a function of $\langle\sigma v\rangle$, determining the minimum $\chi^{2}$, and then set $95 \%$ exclusion limits on $\langle\sigma v\rangle$ from the condition $\Delta \chi^{2}=3.84$. Formally, the correct procedure would amount to fixing $m_{\mathrm{DM}}$ to a grid of values and to performing a separate fit for each of these values. However, such a procedure would be computationally very demanding and would lead to results very similar to those obtained using the 1D profile likelihood for slices in $m_{\mathrm{DM}}$. This is shown in Fig. 3, comparing the black line with the three black dots, which are the limits derived with the formally accurate procedure for the three values of $m_{\mathrm{DM}}$.

In order to obtain an estimate of the systematic uncertainties affecting the limits, we perform fits with different diffusion models, rigidity cuts, DM profiles, and antiproton production cross sections. The various limits are shown in Fig. 3. Not surprisingly, the worst limits are obtained when fixing the diffusion zone height $z_{h}$ to the minimal considered value of $2 \mathrm{kpc}$, since in this case a large fraction of a potential DM signal outside the diffusion zone cannot reach Earth. Correspondingly, setting the diffusion zone height to the maximal value we consider, $z_{h}=7 \mathrm{kpc}$, leads to a larger DM contribution and thus stronger constraints. Neglecting convection in the diffusion equation and/or changing the DM profile from NFW to Burkert does not have a significant impact on the fit.

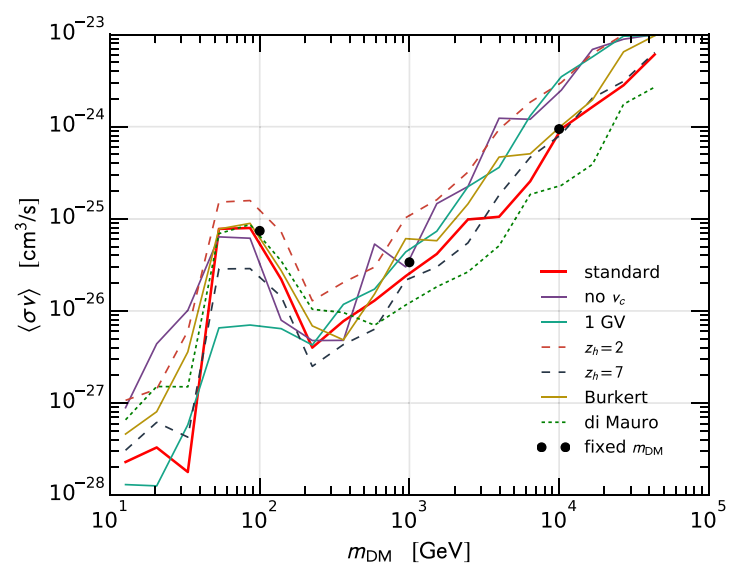

FIG. 3. Limits on the DM annihilation cross section into $b \bar{b}$ final states for our standard setting, for different diffusion zone heights $z_{h}$, for propagation without convection, for an alternative antiproton cross-section model, for the Burkert DM profile, and for rigidities down to $1 \mathrm{GV}$, respectively. We also show limits for three fixed DM masses, as discussed in the text.

The most prominent feature in Fig. 3 is the weak exclusion near DM masses of $80 \mathrm{GeV}$, where the fit prefers a significant DM component. The exclusion becomes much stronger for a fit down to low rigidities of $1 \mathrm{GV}$, which also disfavors a DM signal. However, as argued above, the simple force-field approximation is not expected to describe well solar modulation at rigidities $R \lesssim 5 \mathrm{GV}$, and more work is needed to interpret the low rigidity data in a reliable way.

We have emphasized the importance of the antiproton production cross section for a reliable estimate of the antiproton flux. Adopting the more recent cross-section model from Ref. [42], rather than the GALPROP default [41], has little impact on the fit near $m_{\mathrm{DM}} \approx 80 \mathrm{GeV}$, but the different energy dependence of the cross-section models leads to a change in the DM limits for light and heavy DM.

In Fig. 4, we summarize the result of our fit and show both the evidence for a DM component in the $\mathrm{CR}$ antiproton flux as well as limits on the DM annihilation cross section. The systematic uncertainty on the exclusion limit is shown as an uncertainty band obtained from the envelope of the various fits presented in Fig. 3. In our baseline scenario (solid line), we can exclude thermal DM with $\langle\sigma v\rangle \approx 3 \times 10^{-26} \mathrm{~cm}^{3} \mathrm{~s}^{-1}$ annihilating into $b \bar{b}$ for $\mathrm{DM}$ masses below about $50 \mathrm{GeV}$ and in the range between approximately 150 and $1500 \mathrm{GeV}$. Even considering our most conservative propagation scenario, we achieve strong limits and can exclude thermal DM below about $50 \mathrm{GeV}$ and in the range between approximately 150 and $500 \mathrm{GeV}$. The results for other hadronic annihilation channels, and for annihilation into $Z Z$ and $W^{+} W^{-}$final states, are very similar; in Supplemental Material [28], we provide limits for DM annihilation into $W^{+} W^{-}$as a further explicit example.

In comparison with the results derived in Ref. [50], from gamma-ray observations of nearby dwarf galaxies, we improve the annihilation cross-section limits by a factor 


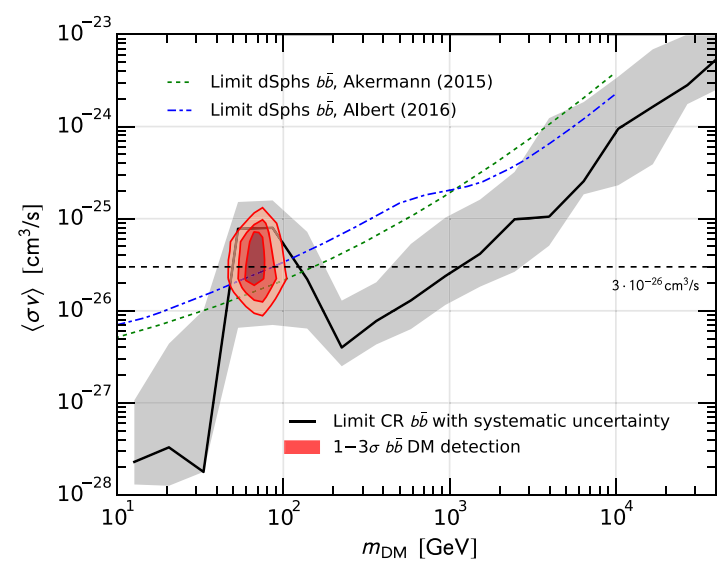

FIG. 4. Best fit regions $(1,2$, and $3 \sigma)$ for a DM component of the antiproton flux and limits on the DM annihilation cross section into $b \bar{b}$ final states. The gray shaded uncertainty band is obtained from the envelope of the various fits presented in Fig. 3. For comparison, we show limits on the annihilation cross section obtained from gamma-ray observations of dwarf galaxies $[50,51]$ and the thermal value of the annihilation cross section, $\langle\sigma v\rangle \approx 3 \times 10^{-26} \mathrm{~cm}^{3} \mathrm{~s}^{-1}$.

of $\sim 4$ for all DM masses except those around $80 \mathrm{GeV}$. We also see from Fig. 4 that, similarly to the DM interpretation of the Galactic center gamma-ray excess, the preferred region of a DM signal in the antiproton flux is in tension with the dwarf galaxy constraints. However, this tension can be relieved with a more conservative estimate of the DM content of the dwarf galaxies [52]. Also, a recent analysis using newly discovered dwarf galaxies [51] actually provides weaker limits, also shown in Fig. 4, further relieving the tension.

Summary and conclusion.-In conclusion, the very accurate recent measurement of the $\mathrm{CR}$ antiproton flux by the AMS-02 experiment allows us to achieve unprecedented sensitivity to possible DM signals, a factor of $\sim 4$ stronger than the limits from gamma-ray observations of dwarf galaxies.

Furthermore, we find an intriguing indication for a DM signal in the antiproton flux, compatible with the DM interpretation of the Galactic center gamma-ray excess. A deeper examination of such a potential signal would require a more accurate determination of the antiproton production cross section, to constrain the flux of secondary antiprotons, as well as an accurate modeling of solar modulation at low rigidities of less than about $5 \mathrm{GV}$.

We thank Jan Heisig, Julien Lesgourgues, Stefan Schael, and Pasquale Serpico for helpful discussions and comments.

Note added.-Recently, we became aware of a similar work [53]. They perform an analysis using methodologies analogous to the ones of this Letter and find results consistent with ours. *cuoco@physik.rwth-aachen.de †mkraemer@physik.rwth-aachen.de *korsmeier@physik.rwth-aachen.de

[1] L. Bergstrom, J. Edsjo, and P. Ullio, Astrophys. J. 526, 215 (1999).

[2] F. Donato, N. Fornengo, D. Maurin, P. Salati, and R. Taillet, Phys. Rev. D 69, 063501 (2004).

[3] T. Bringmann and P. Salati, Phys. Rev. D 75, 083006 (2007).

[4] F. Donato, D. Maurin, P. Brun, T. Delahaye, and P. Salati, Phys. Rev. Lett. 102, 071301 (2009).

[5] N. Fornengo, L. Maccione, and A. Vittino, J. Cosmol. Astropart. Phys. 04 (2014) 003.

[6] D. Hooper, T. Linden, and P. Mertsch, J. Cosmol. Astropart. Phys. 03 (2015) 021.

[7] V. Pettorino, G. Busoni, A. De Simone, E. Morgante, A. Riotto, and W. Xue, J. Cosmol. Astropart. Phys. 10 (2014) 078.

[8] M. Boudaud, M. Cirelli, G. Giesen, and P. Salati, J. Cosmol. Astropart. Phys. 05 (2015) 013.

[9] J. A. R. Cembranos, V. Gammaldi, and A. L. Maroto, J. Cosmol. Astropart. Phys. 03 (2015) 041.

[10] M. Cirelli, D. Gaggero, G. Giesen, M. Taoso, and A. Urbano, J. Cosmol. Astropart. Phys. 12 (2014) 045.

[11] T. Bringmann, M. Vollmann, and C. Weniger, Phys. Rev. D 90, 123001 (2014).

[12] G. Giesen, M. Boudaud, Y. Genolini, V. Poulin, M. Cirelli, P. Salati, and P. D. Serpico, J. Cosmol. Astropart. Phys. 09 (2015) 023.

[13] C. Evoli, D. Gaggero, and D. Grasso, J. Cosmol. Astropart. Phys. 12 (2015) 039.

[14] M. Aguilar et al. (AMS Collaboration), Phys. Rev. Lett. 117, 091103 (2016).

[15] M. Aguilar et al. (AMS Collaboration), Phys. Rev. Lett. 117, 231102 (2016).

[16] G. Johannesson et al., Astrophys. J. 824, 16 (2016).

[17] Q. Yuan, S.-J. Lin, K. Fang, and X.-J. Bi, arXiv:1701.06149 [Phys. Rev. D (to be published)].

[18] H.-B. Jin, Y.-L. Wu, and Y.-F. Zhou, arXiv:1701.02213.

[19] S.-J. Lin, X.-J. Bi, J. Feng, P.-F. Yin, and Z.-H. Yu, arXiv:1612.04001.

[20] M. Korsmeier and A. Cuoco, Phys. Rev. D 94, 123019 (2016).

[21] J. F. Navarro, C. S. Frenk, and S. D. M. White, Astrophys. J. 462, 563 (1996).

[22] P. Salucci, F. Nesti, G. Gentile, and C. F. Martins, Astron. Astrophys. 523, A83 (2010).

[23] A. Burkert, Symp.-Int. Astron. Union 171, 175 (1996) [Astrophys. J. 447, L25 (1995)].

[24] M. Cirelli, G. Corcella, A. Hektor, G. Hutsi, M. Kadastik, P. Panci, M. Raidal, F. Sala, and A. Strumia, J. Cosmol. Astropart. Phys. 03 (2011) 051; 10 (2012) E01.

[25] A. W. Strong, I. V. Moskalenko, and V. S. Ptuskin, Annu. Rev. Nucl. Part. Sci. 57, 285 (2007).

[26] A. W. Strong, I. V. Moskalenko, and O. Reimer, Astrophys. J. 537, 763 (2000); 541, 1109(E) (2000).

[27] A. W. Strong, arXiv:1507.05020.

[28] See Supplemental Material at http://link.aps.org/ supplemental/10.1103/PhysRevLett.118.191102 for further 
details on the theoretical setup and some extended results including the Boron over Carbon ratio.

[29] I. V. Moskalenko, A. W. Strong, J. F. Ormes, and M. S. Potgieter, Astrophys. J. 565, 280 (2002).

[30] M. Aguilar et al. (AMS Collaboration), Phys. Rev. Lett. 114, 171103 (2015).

[31] M. Aguilar et al. (AMS Collaboration), Phys. Rev. Lett. 115, 211101 (2015).

[32] Y. S. Yoon et al., Astrophys. J. 728, 122 (2011).

[33] E. C. Stone, A. C. Cummings, F. B. McDonald, B. C. Heikkila, N. Lal, and W. R. Webber, Science 341, 150 (2013).

[34] F. Feroz, M. P. Hobson, and M. Bridges, Mon. Not. R. Astron. Soc. 398, 1601 (2009).

[35] C. Gordon and O. Macias, Phys. Rev. D 88, 083521 (2013); 89, 049901(E) (2014).

[36] K. N. Abazajian, N. Canac, S. Horiuchi, and M. Kaplinghat, Phys. Rev. D 90, 023526 (2014).

[37] T. Daylan, D. P. Finkbeiner, D. Hooper, T. Linden, S. K. N. Portillo, N. L. Rodd, and T. R. Slatyer, Phys. Dark Univ. 12, 1 (2016).

[38] M. Ajello et al. (Fermi-LAT Collaboration), Astrophys. J. 819, 44 (2016).

[39] F. Calore, I. Cholis, C. McCabe, and C. Weniger, Phys. Rev. D 91, 063003 (2015).

[40] O. Adriani et al. (PAMELA Collaboration), Phys. Rev. Lett. 105, 121101 (2010).
[41] L. C. Tan and L. K. Ng, J. Phys. G 9, 227 (1983).

[42] M. di Mauro, F. Donato, A. Goudelis, and P. D. Serpico, Phys. Rev. D 90, 085017 (2014).

[43] M. Kachelriess, I. V. Moskalenko, and S. S. Ostapchenko, Astrophys. J. 803, 54 (2015).

[44] F. Donato, D. Maurin, P. Salati, A. Barrau, G. Boudoul, and R. Taillet, Astrophys. J. 563, 172 (2001).

[45] C. Evoli, I. Cholis, D. Grasso, L. Maccione, and P. Ullio, Phys. Rev. D 85, 123511 (2012).

[46] R. Kappl and M. W. Winkler, J. Cosmol. Astropart. Phys. 09 (2014) 051.

[47] M. W. Winkler, J. Cosmol. Astropart. Phys. 02 (2017) 048.

[48] I. Cholis, D. Hooper, and T. Linden, Phys. Rev. D 93, 043016 (2016).

[49] C. Corti, V. Bindi, C. Consolandi, and K. Whitman, Astrophys. J. 829, 8 (2016).

[50] M. Ackermann et al. (Fermi-LAT Collaboration), Phys. Rev. Lett. 115, 231301 (2015).

[51] A. Albert et al. (DES, Fermi-LAT Collaboration), Astrophys. J. 834, 110 (2017).

[52] V. Bonnivard et al., Mon. Not. R. Astron. Soc. 453, 849 (2015).

[53] M.-Y. Cui, Q. Yuan, Y.-L. S. Tsai, and Y.-Z. Fan, preceding Letter, Phys. Rev. Lett. 118, 191101 (2017). 